\title{
BIBLIOGRAFÍA Y RECURSOS ELECTRÓNICOS \\ PARA LA TRADUCCIÓN MULTIMEDIA, LA TRADUMÁTICA \\ Y LA ENSEÑANZA DE LENGUAS \\ $M^{a}$ Isabel Martinez Robledo \\ Universidad de Granada
}

\section{ABSTRACT}

This bibliographic article collects a series of bibliographical and electronic resources intended for multimedia translation, tradumatics and online languages teaching, particularly French, English and Italian.

KEYWORDS: Bibliography, electronic resources, multimedia translation, tradumatics, languages teaching, localization.

\section{RESUMEN}

El presente artículo bibliográfico recoge una serie de recursos bibliográficos y electrónicos para la Traducción multimedia, la Tradumática y la Enseñanza de Lenguas online, principalmente el francés, el inglés y el italiano.

PALABRAS CLAVE: Bibliografía, recursos electrónicos, Traducción multimedia, Tradumática, Enseñanza de Lenguas, Localización.

\section{INTRODUCCIÓN}

Este artículo bibliográfico presenta un repertorio comentado de bibliografía especializada y recursos electrónicos centrado en las nuevas tecnologías orientadas a los campos de la Traducción y de la Enseñanza de Lenguas: la fusión de la Informática con la Traducción y con la Lingüística, da lugar al nacimiento de nuevas disciplinas y subdisciplinas como la Traducción multimedia, la Tradumática, la Terminótica, la Localización o el E-Learning. Para englobar todos estos contenidos, este artículo se divide en cuatro secciones:

- Referencias bibliográficas: libros, capítulos de libros y artículos de revistas.

- Recursos electrónicos para la Traducción multimedia. 
- Recursos electrónicos para la Tradumática, la Terminótica y la Localización.

- Recursos electrónicos para la Enseñanza de Lenguas: especialmente francés, inglés e italiano.

\section{REFERENCIAS BIBLIOGRÁFICAS}

Amat, N. (1990): La biblioteca electrónica. Barcelona, Fundación Germán Sánchez. Obra en español sobre las características de las bibliotecas electrónicas.

Bello, P., Feria, A. y Ferrán, J. M. (1998): Didáctica de las segundas lenguas. Estrategias y recursos básicos. Madrid, Santillana.

Obra que realiza un análisis de los principales recursos y estrategias que existen a la hora de llevar a cabo el proceso de enseñanza-aprendizaje de las segundas lenguas.

BOU BAUZÁ, G. (2003): El guión multimedia. Madrid, Anaya Multimedia.

Manual que incluye las principales técnicas para la creación de guiones multimedia basadas en la Narración Audiovisual Interactiva para el diseño de productos multimedia.

Bou BauzÁ, G., Cascudo, C. T. y Borén, L. H. (2003): e-learning. Madrid, Anaya Multimedia.

Manual que incluye una introducción a los conceptos sobre el e-learning y la enseñanza a través de Internet.

Boyle, T. (1997): Design for Multimedia Learning. Hertfordshire, Prentice Hall.

Manual en inglés sobre el aprendizaje multimedia, que trata temas como el diseño conceptual, el diseño de la presentación y el desarrollo, evaluación y distribución de proyectos multimedia para la enseñanza.

Burrows, T. (1999): The text in the machine: electronic texts in the Humanities, Nueva York.

Obra en inglés sobre el tratamiento electrónico de textos y la edición de textos en el campo de Humanidades.

CARIDAD, M. Y Moscoso, P. (1991): Los sistemas hipertexto e hipermedios. Una nueva aplicación en informática documental. Madrid, Cátedra.

Obra en español sobre la aplicación de los sistemas de hipertexto e hipermedia a la edición electrónica de textos. 
CıOTTI, F. (1995): “Testi elettronici e banche dati testuali: probleme teorici e tecnologie" en Schede umanistiche, Nuova Serie, 2.

Obra en italiano sobre la problemática teórica y tecnológica del tratamiento de textos electrónicos y los bancos de datos textuales.

CHARPIN, F. (1994): “Traitement informatique de l'ordre de mots" en Techniques et méthodologies modernes appliquées à l'antiquité, Laboratoire LITALA, 1, pp. 75-107. Artículo en francés sobre el tratamiento informático de los textos y el orden de las palabras.

Del Moral, E. (1997): "Diseño de hiperdocumentos y su repercusión en el aprendizaje" en Actas del Congreso Internacional de Informática Educativa 1997. Madrid, UNED.

Artículo en español que trata sobre el diseño de documentos de hipertexto destinados a la enseñanza.

Díaz, P., Catenazzi, N. y Aedo, I. (1996): De la multimedia a la hipermedia. Madrid, Ra.ma.

Obra en español que analiza las tecnologías multimedia e hipermedia orientadas al tratamiento y edición electrónica de textos.

Díaz de Bustamante, J. M. (1986): "De tratamiento de textos a bases de datos. Unas posibilidades para las investigaciones filológicas" en Euphrosyne, 14, pp. 183-196.

Artículo en español que trata sobre la investigación del tratamiento electrónico de textos y las bases de datos aplicada a la Filología.

EL SADDIK, A. (2001): Interactive Multimedia Learning. Shared reusable visualisationbased modules. New York, Springer.

Manual en inglés que introduce los conceptos más relevantes respecto al desarrollo y la reusabilidad de contenido multimedia en los sistemas de enseñanza-aprendizaje a través de Internet.

England, E. y Finney, A. (1999): Managing Multimedia. Project Management for Interactive Media. Essex, Addison-Wesley.

Manual en inglés para el diseño, la producción y la programación de productos multimedia que incluye de forma detallada todas las fases, desde la definición hasta la conclusión del proyecto.

Escudero, J. M. (1979): Tecnología educativa: diseño de material escrito para la enseñanza. Valencia, Nau Llibres. 
Obra en español que trata sobre el diseño de material escrito orientado a la enseñanza y a la tecnología educativa.

FABER, P. y JimÉnEZ, C. (eds.) (2002): Investigar en Terminología. Granada, Editorial Comares Interlingua.

Volumen que trata los fundamentos teóricos de la metodología de la investigación terminológica, la terminología basada en el corpus y las aplicaciones prácticas del grupo ONCOTERM: Sistema bilingüe de información y recursos oncológicos.

Ferrés, J. y MARquès Graells, P. (coords.) (1996-2003): Comunicación Educativa y Nuevas Tecnologías. Barcelona, Editorial Praxis.

Obra en español que trata sobre el uso de las nuevas tecnologías para el tratamiento y la edición electrónica de textos orientados a la comunicación educativa.

FuCHS, C. (1993): Linguistique et traitements automatiques des langues. Baume-lesDames, Hachette Supérieur.

Obra en francés que se centra en el tratamiento automático de las lenguas, tanto en los niveles de tratamiento (fonética y fonología, prosodia, morfología, sintaxis y semántica) como en los dominios de tratamiento (tratamiento de la palabra, traducción automática, comprensión automática de textos, generación automática de textos y diálogo hombre-máquina).

GARCíA, F. (2000): "La narrativa hipermedia aplicada a la educación compartida entre el autor y el lector, entre el profesor y el alumno" en PÉREZ, R. (coord.) Redes multimedia y diseños virtuales. Oviedo, Dpto. Educación de la Universidad de Oviedo.

Artículo en español que trata sobre las técnicas de narración hipermedia aplicadas a educación.

GARrigues, M. (1988): "Nouvelles technologies et apprentissage des langues" en Le Français dans le Monde. Recherches et Applications, Numéro Spécial AoûtSeptembre. Paris.

Número especial de la publicación francesa Le Français dans le Monde dedicado a las nuevas tecnologías y el aprendizaje de lenguas. Los principales temas que trata son la Enseñanza asistida por Ordenador, la conjunción entre la Informática y la Didáctica, los tipos de software empleado para la enseñanza, la videoconferencia y la telemática.

Grishman, R. (1991): Introducción a la Lingüistica Computacional. Madrid, Visor Lingüística y Conocimiento. 
Obra que realiza una introducción al concepto de Lingüística Computacional y a los distintos tipos de análisis: análisis sintáctico, análisis semántico, análisis del discurso y generación del lenguaje, mediante la descripción de los tipos de gramáticas, parsers y analizadores que se emplean en este campo.

LOWE, D. y Hall, W. (1999): Hypermedia and the web. An engineering approach. Chichester, Wiley.

Manual en inglés que analiza la estructuración, las características y los recursos de la información interactiva y los documentos de hipertexto tanto en Internet como en CD-ROM.

Marcelo, C. et al. (2002): e-learning Teleformación. Diseño, Desarrollo y Evaluación de la Formación a través de Internet. Barcelona, Ediciones Gestión 2000.com.

Obra que realiza una introducción al concepto de e-learning y teleformación, así como al diseño, desarrollo y evaluación del proceso de enseñanza-aprendizaje a través de Internet mediante aplicaciones informáticas.

Montalt i ResurRecció, V. (2003): “La traducción de géneros electrónicos: el caso de la localización" en Panorama Actual de la Investigación en Traducción e Interpretación. Volumen II, 313-328. Granada, Editorial Atrio.

Artículo que trata sobre el género de la localización como futuro de los traductores especializados del siglo XXI.

Moreno Sandoval, A. (1998): Lingüistica Computacional. Madrid, Editorial Síntesis.

Obra que trata sobre la delimitación del campo de la Lingüística Computacional, el panorama general de esta disciplina y los distintos modelos que existen: simbólicos, probabilísticos e inspirados en la Biología (redes neuronales).

Nielsen, J. (1990): Hypertext and bypermedia. Boston, Academic Press.

Obra en inglés sobre las tecnologías de hipertexto e hipermedia para la edición de documentos electrónicos.

Orihuela, J. L. y SANTOS, M. L. (1999): Introducción al Diseño Digital. Concepción y Desarrollo de Proyectos de Comunicación Interactiva. Madrid, Anaya Interactiva.

Obra en español que realiza una introducción al diseño digital orientado al desarrollo de proyectos de comunicación interactiva.

RuEDA, R. (1997): Hipertexto: Representación y aprendizaje. Bogotá, Tecné.

Obra en español sobre la función y el uso del hipertexto en el aprendizaje. 
Ortega Carrillo, J. A. (1997): Comunicación Visual y Tecnología Educativa. Granada, Grupo Editorial Universitario.

Obra que trata sobre la evolución histórica y la naturaleza de la Comunicación Visual, las disciplinas de la percepción visual (semiótica, morfosintaxis y semántica) y su relación con la tecnología educacional, es decir, las implicaciones organizativas de las nuevas tecnologías aplicadas a la formación y la creación de materiales multimedia.

PÉrez Torres, M. I. (2001): "La enseñanza de las lenguas y las nuevas tecnologías" en Actas de La Enseñanza de Lenguas en el Nuevo Milenio, 69-77. Granada, Grupo Editorial Universitario.

Artículo que trata sobre la incorporación de las nuevas tecnologías en la enseñanza de lenguas con la llegada de la denominada "Sociedad de la Información": recursos audiovisuales, materiales multimedia e Internet.

SALINAS, J. (1994): "Hipertexto e hipermedia en la enseñanza universitaria", Pixel-Bit: revista de medios de educación, $n^{0} 1$, pp. 15-29.

Artículo en español sobre la introducción de las tecnologías de hipertexto e hipermedia en la enseñanza universitaria.

SHaddock, P. (1994): Creaciones Multimedia. Madrid, Anaya Multimedia.

Manual para la creación de catálogos electrónicos, programas educativos interactivos, películas digitales en tiempo real y vídeos musicales, incluyendo todas las fases, desde el diseño hasta la postproducción.

TEBÉ, C. (2003): "Las herramientas informáticas en la didáctica de la traducción especializada" en Panorama Actual de la Investigación en Traducción e Interpretación. Volumen II, 329-341. Granada, Editorial Atrio.

Artículo sobre las herramientas informáticas que sirven de ayuda al traductor especializado, como aquéllas que permiten la integración de la traducción y la terminología en la Memoria de Traducción o aplicaciones de Traducción Asistida por Ordenador.

Tormo, R. (2000): "Uso de hipertextos para facilitar el aprendizaje en la universidad", Revista Comunicación y Pedagogía, núm. 168, pp. 65-68 y Revista Comunicación y Pedagogía, núm. 169, pp.22-27.

Artículo en español sobre la introducción de las tecnologías de hipertexto e hipermedia en la enseñanza universitaria.

WodAski, R. (1996): La Biblia de Multimedia. Madrid, Anaya Multimedia.

Manual de uso de los componentes principales de la tecnología multimedia: imágenes, animaciones, sonido, vídeo, fotografía y texto. Este libro enseña a 
crear y modificar sonidos, a utilizar las herramientas gráficas más potentes del mercado, a programar aplicaciones multimedia, a desarrollar presentaciones interactivas y a crear animaciones y vídeos.

ZARANDiETA MORÁN, F. y ZARANDIETA MORÁN, J. A. (2002): La educación por Internet. Edición 2003. Madrid, Anaya Multimedia.

Obra que trata sobre el concepto y las nociones básicas de e-learning o teleformación, es decir, de la formación a través de Internet.

\section{RECURSOS ELECTRÓNICOS PARA LA TRADUCCIÓN MULTIMEDIA}

http://www.webpersonal.net/dmarques/resources/soft.htm

Lost in Babel. Enlaces con recursos electrónicos para los traductores: diccionarios bilingües y monolingües, enciclopedias, bases de datos terminológicas, recursos para la localización, etc.

http://www.multingles.net/traduccion.htm

Selección de recursos electrónicos para los traductores: diccionarios, traductores automáticos, servicios de traducción, glosarios, diccionarios de jergas y expresiones, etc.

http://www.ucm.es/info/multidoc/multidoc/revista/index.htm

Cuadernos de Documentación Multimedia. Artículos sobre Multimedia y Nuevas Tecnologías.

http://algomasquetraducir.com/

Blog sobre traducción profesional, localización de videojuegos y tecnologías de la traducción.

\section{RECURSOS ELECTRÓNICOS PARA LA TRADUMÁTICA, LA TERMINÓTICA Y LA LOCALIZACIÓN}

http://www.trados.com/

Información sobre todos los productos de la gama Trados/MultiTerm, incluida la versión demo del software líder del mercado en Traducción Asistida por Ordenador y Gestión de Terminología. 
http://www.atril.com/

Información sobre todos los productos de la compañía Atril, incluida la versión demo del software DéjàV $u$ de Traducción Asistida por Ordenador.

http://www.star-solutions.net/

Información sobre todos los productos de la compañía Star, incluida la versión demo del software Transit de Traducción Asistida por Ordenador.

http://www.fti.uab.es/tradumatica/revista/

Revista «Tradumàtica», traducción y TI (Universitat Autònoma de Barcelona).

http://www.lisa.org/

LISA, Localization Industry Standards Association.

http://www.gala-global.org/

GALA, Globalization and Localization Association.

http://www.wordbank.com/sp/about_us/core_skills.html\#tools

Información sobre herramientas de localización y distintos productos de la compañía Wordbank: traducción, localización, composición tipográfica y edición en múltiples idiomas, creación y gestión de bases de datos terminológicas y memorias de traducción.

http://www.interproinc.com/es/index.asp

Interpro Translation Solutions. Localización de software.

http://www.hispanicoweb.miarroba.com/

Hispánico Web. Selección de software, ficheros de ayuda y parches en español. Tutorial para traducir programas.

http://www.lingoware.com/spanish/index.html

LingoWare. Traduce cualquier aplicación de software o Internet mediante un simple arrastre.

http://www.esteam.gr/

ESTeam. ESTeam Translator integra en un solo producto las Memorias de Traducción y la Traducción Automática. Traducción entre cualquier combinación de lenguas de la Unión Europea, además del Noruego.

http://www.samlight.com/ev/eng/

EV-soft. Herramientas de Traducción Automática: Chino/Inglés. 
http://tranexp.com/

InterTran \& NeuroTran. Herramientas de Traducción Automática: InterTran: cualquier combinación entre Búlgaro, Croata, Checo, Danés, Neerlandés, Inglés, Finlandés, Francés, Alemán, Griego, Húngaro, Islandés, Italiano, Japonés, Noruego, Polaco, Portugués, Portugués (Brasileño), Rumano, Ruso, Serbio, Esloveno, Español (Europeo), Español (Latinoamericano), Sueco y Galés. NeuroTran: Alemán $<=>$ Inglés, Francés $<=>$ Inglés, Español $<=>$ Inglés, Húngaro $<=>$ Inglés y Polaco $<=>$ Inglés, Croata $<=>$ Inglés, Bosnio $<=>$ Inglés, Serbio $<=>$ Inglés.

http://www.wordbank.com/sp/about_us/core_skills.html\#tools Información sobre herramientas de localización y distintos productos de la compañía Wordbank: traducción, localización, composición tipográfica y edición en múltiples idiomas, creación y gestión de bases de datos terminológicas y memorias de traducción.

\section{RECURSOS ELECTRÓNICOS PARA LA ENSEÑANZA DE LENGUAS}

http://www.lepointdufle.net/frances.htm

Ejercicios y recursos de francés.

http://www.francofil.net/es/fle_esp.html

FRANCOFIL. Recursos para profesores de francés lengua extranjera.

http://www.cilf.org/

Conseil International de la Langue Française. Lengua francesa.

http://www.inalf.fr/cgi-bin/inalf.exe (RETOCAR)

L'Institut National de la Langue Française. Lengua francesa.

http://www.cnrs.fr/

Centre National de la Recherche Scientifique. Lengua francesa.

http://platea.pntic.mec.es/ cvera/ressources/recursosfrances.htm

Ressources didactiques sur Internet. Carmen Vera Pérez.

http://www.educa.madrid.org/web/eoi.jesusmaestro.madrid/francesnet.html Recursos de lengua francesa. Escuela Oficial de Idiomas. Aula de Autoaprendizaje. 
http://www.aprenderfrances.org/esfrench/products.asp

Recursos para el aprendizaje de la lengua francesa.

http://www.educaguia.com/Software/frances.asp

Software educativo de francés.

http://www.iicbelgrado.esteri.it/IIC_Madrid/Menu/Imparare_Italiano/Studia re_Italiano_on_line/

Istituto Italiano di Cultura de Madrid. Recursos y exámenes oficiales de lengua italiana.

http://alphalinguistica.sns.it/

Scuola Normale Superiore. Laboratorio de Lingüística. Lengua italiana.

http://alcorcon2006.canalblog.com/archives/recursos_para_italiano/index.ht $\mathrm{ml}$

Aprendizaje de Idiomas y TIC. Lengua italiana.

http://www.favole.org/

Las TIC en clase de lenguas. Lengua italiana.

http://www.cursoitaliano.org/

Curso de lengua italiana.

http://www.english-portal.com/

Noticias y artículos, inglés general y de negocios, ejercicios, gramática, vocabulario, tests, foros...

http://www.mansioningles.com/

Libros, lecturas, programas, cursos, magazine, gramática, ejercicios, práctica, canciones, recursos, profesores, chat...

http://esl.about.com/

Recursos, boletín y noticias, educación bilingüe, EAO, software ESL, gramática, pronunciación, fuentes de consulta, trabajo, formación de profesores...

http://www.iol.org.uk/

Institute of Linguists. Lengua inglesa.

http://www.e-ducativa.com/

E-ducativa. Información sobre las nuevas tecnologías y la educación. 
http://www.educaweb.com/

Educaweb. Información sobre las nuevas tecnologías y la educación.

http://edutec.rediris.es/

Edutec. Información sobre las nuevas tecnologías y la educación.

http://www.multingles.net/articulos.htm

Multinglés. Información sobre el e-learning y la educación. 